\title{
Cross-cultural similarities and differences in North Americans' geographic location judgments
}

\author{
ALINDA FRIEDMAN \\ University of Alberta, Edmonton, Alberta, Canada \\ DENNIS D. KERKMAN \\ Park University, Parkville, Missouri \\ NORMAN R. BROWN \\ University of Alberta, Edmonton, Alberta, Canada \\ DAVID STEA \\ Texas State University, San Marcos, Texas \\ and \\ HECTOR M. CAPPELLO \\ Universidad Nacional Autónoma de México, Cuernavaca, Mexico \\ and Universidad Autónoma de Tamaulipas, Victoria, Tamaulipas, Mexico
}

\begin{abstract}
We examined some potential causes of bias in geographic location estimates by comparing location estimates of North American cities made by Canadian, U.S., and Mexican university students. All three groups placed most Mexican cities near the equator, which implies that all three groups were influenced by shared beliefs about the locations of geographical regions relative to global reference points. However, the groups divided North America into different regions and differed in the relative accuracy of the estimates within them, which implies that there was an influence of culture-specific knowledge. The data support a category-based system of plausible reasoning, in which biases in judgments are multiply determined, and underscore the utility of the estimation paradigm as a tool in cross-cultural cognitive research.
\end{abstract}

The manner in which culture affects cognition poses a rich and complex set of research issues (Bailenson, Shum, Atran, Medin, \& Coley, 2002; Medin, Ross, Atran, Burnett, \& Blok, 2002). In the present article, we examine some of these in the context of judgments about global geography. Specifically, we address how some biases in global location judgments can be attributed to the categorical nature of geographical representations and the processes that use them, whereas others are attributable to cultural asymmetries in geographical knowledge.

Our previous research with Canadian participants (Friedman \& Brown, 2000a, 2000b; Friedman, Brown, \& McGaffey, 2002) indicated that their location estimates for cities in the old and new worlds were based on a categorydriven system of plausible reasoning (Collins \& Michal-

This research was supported by grants from the Natural Sciences and Engineering Research Council of Canada and by Grants DUE-9551939 and 9906418 from the U.S. National Science Foundation. We express our thanks to the students who participated. Correspondence concerning this article should be sent to A. Friedman, Department of Psychology, University of Alberta, Edmonton, AB, T6G 2E9 Canada (e-mail: alinda (a) ualberta.ca). ski, 1989). Geographic categories were psychologically distinct regions that could be independently influenced by new information. Some countries had more than one region, and some regions comprised either one or several countries. The plausible reasoning framework assumes that biases in judgments about global locations are multiply determined because they are influenced by accurate and inaccurate beliefs about geographic regions acquired over the lifespan from a variety of sources.

The key features of the data (Friedman \& Brown, 2000a, 2000b) were that (1) Canadian participants divided North America into four distinct regions (Canada, the northern U.S., the southern U.S., and Mexico), (2) there were usually large boundary zones (gaps) between regions, (3) there was little north-south discrimination among the estimates within most regions, and (4) the estimates became more biased as the cities' actual locations were farther south. Indeed, the average location estimate for most Mexican cities was near the equator, which was an error of approximately 1,500 miles.

These four observations are consistent with the influence of categorical information on location estimates (Brown, 2002; Friedman \& Brown, 2000a, 2000b; Huttenlocher, Hedges, \& Duncan, 1991; McNamara, 1986; 
Stevens \& Coupe, 1978). For example, once cities are assigned to superordinate regions that cover mutually exclusive portions of the response range, they inherit the superordinates' ordinal and metric properties. Furthermore, when item knowledge is sparse, people may rely on prototypes for their estimates, so their within-region response range tends to be truncated. Finally, finding that estimation bias increases as a function of increasing distance from the participants' home region (Canada) appears, at first, to be explained well by factors related to physical proximity: As distance to a target location increases, so does uncertainty and unfamiliarity and, hence, bias (Golledge, 1993; Huttenlocher et al., 1991; Lundberg \& Ekman, 1973; McNamara \& Diwadkar, 1997). However, we disconfirmed the physical proximity hypothesis in a comparative study of Canadian and American participants who lived roughly 1,500 and 250 miles north of the Mexican border, respectively (Friedman, Kerkman, \& Brown, 2002). The participants from Edmonton, Alberta $\left(54^{\circ} \mathrm{N}, 113^{\circ} \mathrm{W}\right)$ underestimated the location of Mexican cities by an average of about $22^{\circ}$. Rather than being more accurate, the participants from San Marcos, Texas $\left(30^{\circ} \mathrm{N}\right.$, $98^{\circ} \mathrm{W}$ ) underestimated the same cities by an additional $10^{\circ}$ (700 miles) farther south. Thus, relative proximity to Mexico could not have been the primary cause of the amount and direction of bias in the Mexican estimates. Pinpointing the source(s) of this bias was the main goal of the present study. To do this, we compared the previously reported data from Albertans and Texans with new data from university students living in Ciudad Victoria, Tamaulipas $\left(24^{\circ} \mathrm{N}, 97^{\circ} \mathrm{W}\right)$, which is approximately 190 miles south of the U.S.-Mexican border.

We adopted the logic of the triangulation strategy described by Bailenson et al. (2002). Their general idea is to use observations from a third group in order to get some leverage on the understanding of similarities and differences between two initial groups. If the third group performs similarly to one of the initial groups, but not to the other, the shared variables between the two similar groups have potential explanatory validity. Equally, any observed differences between two similar groups cannot be attributed to these shared factors; nor can similarities in the performance among all three groups be attributed to them.

The Texans and Tamaulipans lived roughly the same distance from the Mexican border and thus shared a geography and climate not shared by the Albertans. By the triangulation strategy, therefore, neither local geography, climate, nor distance from the U.S.--Mexican border could be responsible for any similarities observed in the estimates across all three groups or for any differences between the Texans and Tamaulipans. To preview, the data we will report provide evidence for two sources of bias in geographic location estimates. The first affected all three groups similarly, because it derives from the categorical nature of geographical representations and the utility and ubiquity of plausible reasoning processes in estimation tasks. The second source of bias was attributable to the presumed a priori differences between the groups in item- level knowledge and to our hypothesized reasons for culturally based asymmetries in such knowledge.

With respect to the first source of bias, the striking similarity observed between the Albertans and the Texans in the degree to which their Mexican estimates were too far south suggested that they used a common strategy and had common (and largely incorrect) metric assumptions. Previous evidence has shown that people use an ordinal conversion strategy when asked to provide numeric estimates in knowledge domains for which the relevant facts are scarce (Brown, 2002; Brown \& Siegler, 1993). For location estimates, we hypothesized that this strategy involves drawing on beliefs about the ordinal positions of the regions and their relation to global reference points to define the response range and to partition it among the regions. Thus, when participants are told that latitudes run from $+90^{\circ}$ to $-90^{\circ}$ at the poles, with the equator at $0^{\circ}$, they must map their beliefs about the relative north-south locations of the continents and their regions onto this range. Their estimates for each city are then generated by identifying its region and selecting a value within that region's range; if little is known about the items within a region, the estimate might be the prototype location for that region.

Note, however, that ordinal conversion per se does not cause estimation bias; rather, incorrect metric beliefs underlie estimation bias. We hypothesized that the mistaken belief responsible for the southward bias observed in Albertans' and Texans' estimates for Mexican cities was that the equator was the lower bound for North America and the upper bound for South America. In effect, the equator provided the southern boundary of the functional response range, with the result that Mexico as a region was biased far to the south of its actual location. A priori, it was likely that the Tamaulipans, too, would use the ordinal conversion strategy and global reference points such as the equator to define and partition their response range in this task. If so, there should be some similarity in the direction and magnitude of the signed and absolute errors for the Mexican cities among all three groups of participants.

In contrast to shared beliefs about the location of regions relative to global landmarks, there are many a priori differences among the groups that might differentially influence their location estimates; we will focus on one. Albertans and Tamaulipans are both from countries with less economic and political influence in the world arena than the U.S. has. This kind of cultural asymmetry has implications for estimation accuracy, because people from less dominant cultures may know more about the dominant ones than vice versa. That is, the dominant culture's international policies, economic trends, information and entertainment media, and so on may affect people in less dominant cultures more than the reverse.

Support for the influence of cultural asymmetry on geographical location estimates comes from a survey of 18- to 24-year-olds from nine different countries (Canada, France, Germany, Great Britain, Italy, Japan, Mexico, Sweden, and the U.S.; National Geographic, 2002). Over all nine groups, the average percentages of participants 
who correctly located the U.S., Canada, and Mexico on a world map were $90.4 \%, 87.0 \%$, and $73.9 \%$, respectively. The Mexicans were the only group tested who correctly located both Mexico and the U.S. at a high rate $(97 \%$ and $96 \%$, respectively). On the basis of these results for estimating the location of nations, the prediction of cultural asymmetry for the present study was that participants from Canada and Mexico should have relatively accurate estimates for city locations in their own countries and the U.S. but less accurate estimates for locations of cities in the third nation. In contrast, Texans should be accurate about U.S. locations but relatively inaccurate about locations in Mexico and Canada.

The most straightforward way to assess the accuracy of item knowledge is by the within-region rank order correlations between actual and estimated latitude. These correlations ignore any overall regional biases, while permitting an estimate of the degree to which participants know the relative locations of cities within regions. In contrast, mean signed error for each region estimates the amount and direction of any regional biases. Clearly, it is possible to be biased in the placement of a region but accurate in estimating the ordinal locations of the cities within it.

The two constructs outlined here are not mutually exclusive. Indeed, combining them predicts that whereas
Mexican participants should have reasonably good item knowledge for the relative locations of Mexican cities, they might share with the other participants the same category-based strategy for placing the regions relative to global landmarks, resulting in biases observed at the regional level.

\section{METHOD}

\section{Participants}

Fifty-five undergraduates from the Universidad Autónoma de Tamaulipas volunteered to participate. All were native-born Mexicans. Ten were excluded because they did not complete the task or failed to follow the instructions. In Friedman, Kerkman, and Brown (2002), 4 Albertans and 5 Texans were excluded for the same reasons, leaving 46 Albertans, 46 Texans, and 45 Tamaulipans in the final sample.

\section{Stimuli}

All the participants estimated the latitudes of the same 44 cities (see Figure 1); 11 cities were from each of the four subjective regions previously identified (Friedman, Kerkman, \& Brown, 2002).

\section{Procedure}

Texan and Tamaulipan participants were tested in their respective universities' computer laboratories; Albertans were tested in individual rooms. All the participants completed two tasks, using identical procedures. The participants first rated their knowledge of

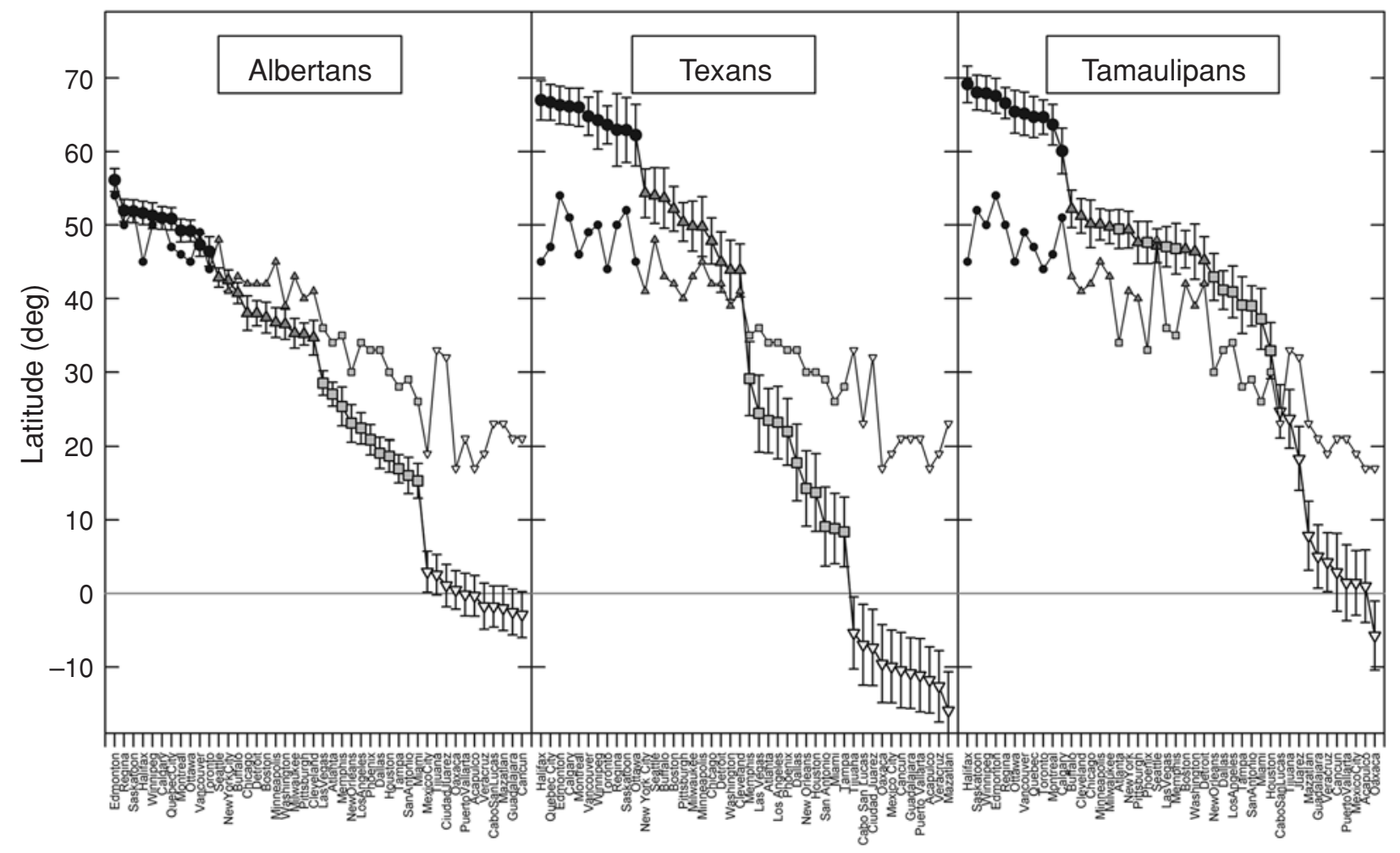

Figure 1. The large symbols are the mean latitude estimates for 44 North American cities by participants from Edmonton, Alberta, San Marcos, Texas, and Ciudad Victoria, Tamaulipas. Cities are rank ordered according to the mean latitude estimate for each group; the city names on the abscissa indicate that order. Error bars are standard errors of the mean for each city across participants within a group. The smaller symbols are the actual latitude of each city. Circles represent Canadian cities, upward-pointing triangles represent northern U.S. cities, squares represent southern U.S. cities, and downward-pointing triangles represent Mexican cities. 
each city on a scale from 0 (no knowledge) to 9 (a lot of knowledge), using the computer's numeric keypad. This familiarized them with the set of stimulus cities. Next, the participants were instructed that the north pole was at $90^{\circ}$ north latitude, the equator was at $0^{\circ}$, and the south pole was at $90^{\circ}$ south. They then estimated the latitude of each city, using the numeric keypad, followed by " $n$ " or "s" for north or south latitude. The order of cities was randomized for each participant and task, and the screen was refreshed after each trial so that the participants could not refer to their previous estimates. Instructions were presented in English for Albertans and Texans and in Spanish ${ }^{1}$ for Tamaulipans.

\section{RESULTS}

We used a $p<.05$ criterion for significance. Figure 1 shows the mean estimates for each city and group. The similarities and differences among the groups provide evidence for an influence of both global reference points and cultural asymmetry on the estimates.

\section{Evidence for the Influence of Global Reference Points}

The most surprising aspect of the data was that the Tamaulipans estimated most Mexican cities to be located near the equator (Figure 1). This error appears to reflect their belief, shared by the Texans and Albertans, that the equator serves as either the southern boundary of the North American range or as the prototype of the Mexican region. The group $\times$ region interactions below primarily reflect differences in the groups' beliefs about the northern boundary of the range. Albertans were relatively accurate, but Texans and Tamaulipans' estimates of Canadian locations were too far north. ${ }^{2}$
An ANOVA on the average estimated latitude for each region yielded a group (Albertans, Texans, Tamaulipans) $\times$ region (Canada, northern U.S., southern U.S., Mexico) interaction $\left[F(6,402)=13.25, M S_{\mathrm{e}}=186.26\right]$. The same interaction was significant for signed and absolute errors $\left[F(6,402)=13.24, M S_{\mathrm{e}}=186.35\right.$, and $F(6,402)=3.12$, $M S_{\mathrm{e}}=117.91$, respectively; see Figure 2]. Signed errors were the estimated minus the actual latitude for each city, averaged over cities within regions. Absolute errors were the absolute value of the signed errors, averaged within regions. Because the three groups differed in their overall range of estimates, we also computed each participant's signed and absolute errors for each city as a percentage of that individual's range of responses; the interactions were still significant for both measures $[F(6,402)=11.80$, $M S_{\mathrm{e}}=98.74$, and $\left.F(6,402)=9.73, M S_{\mathrm{e}}=90.31\right]$. All three groups made large signed and absolute errors on Mexican cities, reflecting shared but mistaken metric beliefs about the cities' equatorial locations. Because all the groups displayed this pattern of bias, neither distance to the border nor shared climate and geography could have been responsible for it.

The actual difference in mean latitude between the Canadian and the U.S. stimulus cities was smaller than the mean difference between the U.S. and the Mexican cities $\left(11.5^{\circ}\right.$ vs. $14.6^{\circ}$, respectively). However, in terms of percentages, the Canadian-U.S. mean difference vs. the U.S.-Mexican mean difference consisted of a $44 \%-56 \%$ split of the total $26.1^{\circ}$ between the Canadian and the Mexican means. If the participants represented this distinction, then when they "stretched" Mexican cities toward the equator, the U.S.-Mexican distance should have stretched
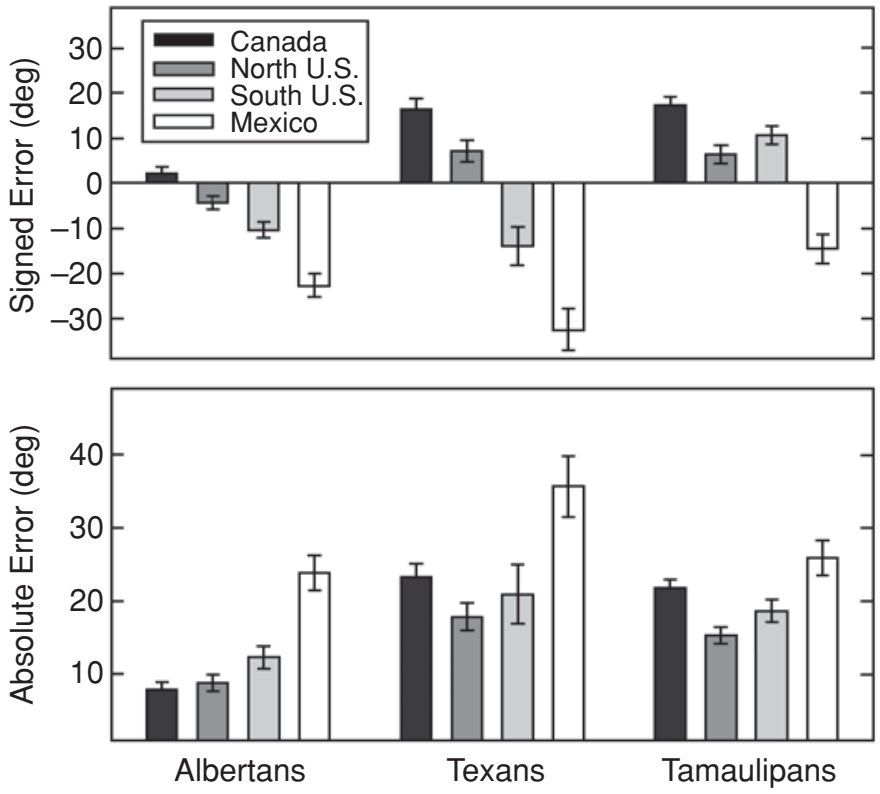

Figure 2. The top panel shows the average signed errors, and the bottom panel shows the average absolute errors for each group and region. Error bars are standard errors of the mean for each region, computed across participants within a group. 
more than the Canadian-U.S. distance, to maintain the relative distances between regional means. ${ }^{3}$

To examine this idea, we obtained the mean difference in latitude estimates between Canada and the U.S. and between the U.S. and Mexico for each participant (Figure 3, top). These were used as the numerators of two ratios; the denominator for both was each participant's CanadaMexico mean estimated difference in latitudes. These ratios show whether the participants had a $44 \%-56 \%$ split between regional means, based on their own scales.

There was a main effect of ratio $[F(1,134)=42.81$, $\left.M S_{\mathrm{e}}=462.53\right]$ : The Canadian-U.S. and the U.S.-Mexican percentages were $43 \%-57 \%$ for the Albertans, $46 \%-54 \%$ for the Texans, and 36\%-64\% for the Mexicans (Figure 3, bottom). The group $\times$ ratio interaction $[F(2,134)=4.47$, $\left.M S_{\mathrm{e}}=462.53\right]$ indicated that the Tamaulipans exaggerated the ratio difference more than did the other two groups; the exaggeration reflects the fact that they were the only group for whom the southern U.S. estimates were too far north. Overall, the ratio data show that the participants scaled North America to accommodate their beliefs about its range, while maintaining approximately correct relative distances between its regions.

\section{Evidence for the Influence of Cultural Asymmetry}

We computed rank order correlations between the actual and the estimated latitudes, separately, for each participant and region. ${ }^{4}$ The Fisher-transformed correlations yielded effects of group $\left[F(2,134)=3.67, M S_{\mathrm{e}}=0.24\right]$ and region $\left[F(3,402)=44.51, M S_{\mathrm{e}}=0.14\right]$ and their interaction $\left[F(6,402)=21.30, M S_{\mathrm{e}}=0.14\right]$. The backtransformed means (Figure 4) support the cultural asymmetry predictions. All the groups had relatively large rank order correlations for their home region, and both Albertans and Tamaulipans had relatively large rank order correlations for the two U.S. regions, in addition to their own. The Texans were relatively inaccurate about the relative locations of both Canadian and Mexican cities. Furthermore, geographical distance cannot wholly explain these results. For example, both Albertans and Tamaulipans had higher correlations for northern U.S. cities than for southern U.S. cities. In addition, in the survey mentioned earlier (National Geographic, 2002), the performance of participants from France, Germany, Great Britain, Italy, Japan, and Sweden also argues against the idea that geographical distance can account for the present pattern of differences between groups. Arguably, these six countries are functionally equidistant to Canada, the U.S., and Mexico, yet the participants from these countries exhibited a pattern for identifying the location of the three North American nations that was similar only to the pattern for the present Canadian participants. In contrast, the Mexicans were the only participants who were accurate in locating Mexico in the National Geographic study and Mexican cities in the present one.

The knowledge ratings also corroborated the cultural asymmetry position (Figure 4). All three groups rated
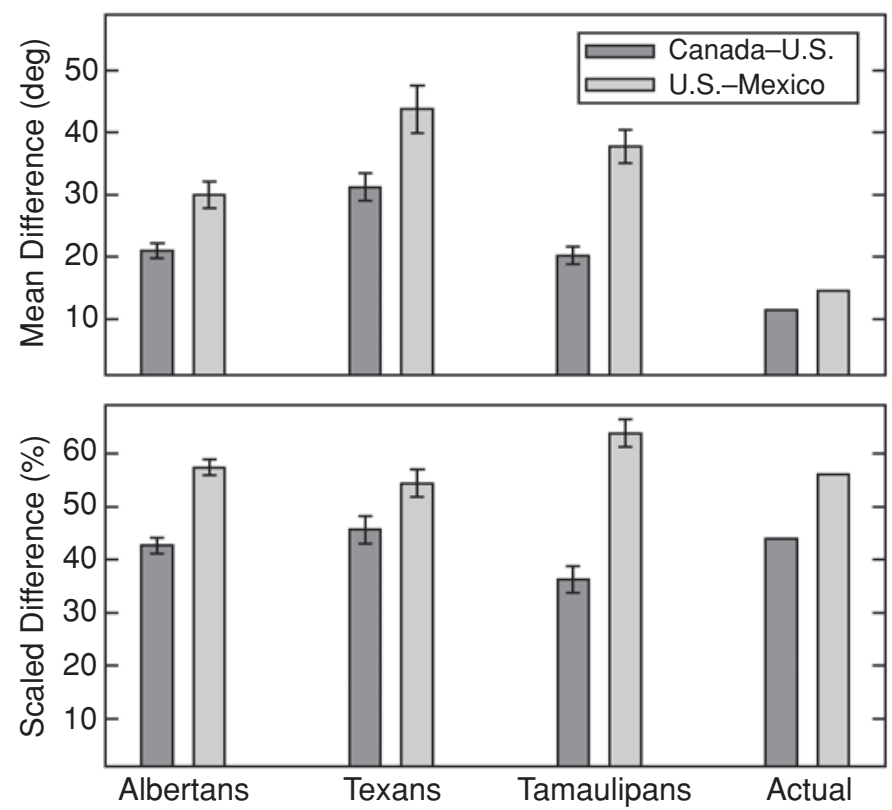

Figure 3. The top panel shows the average difference between the mean estimated latitudes of Canadian and U.S. cities and between the mean estimated latitudes of U.S. and Mexican cities for each participant group. The bottom panel shows the mean estimated differences for each pair of regions, expressed as a percentage of the difference between the means of the Canadian and the Mexican cities for each participant. Error bars are standard errors for each measure, computed across participants. 

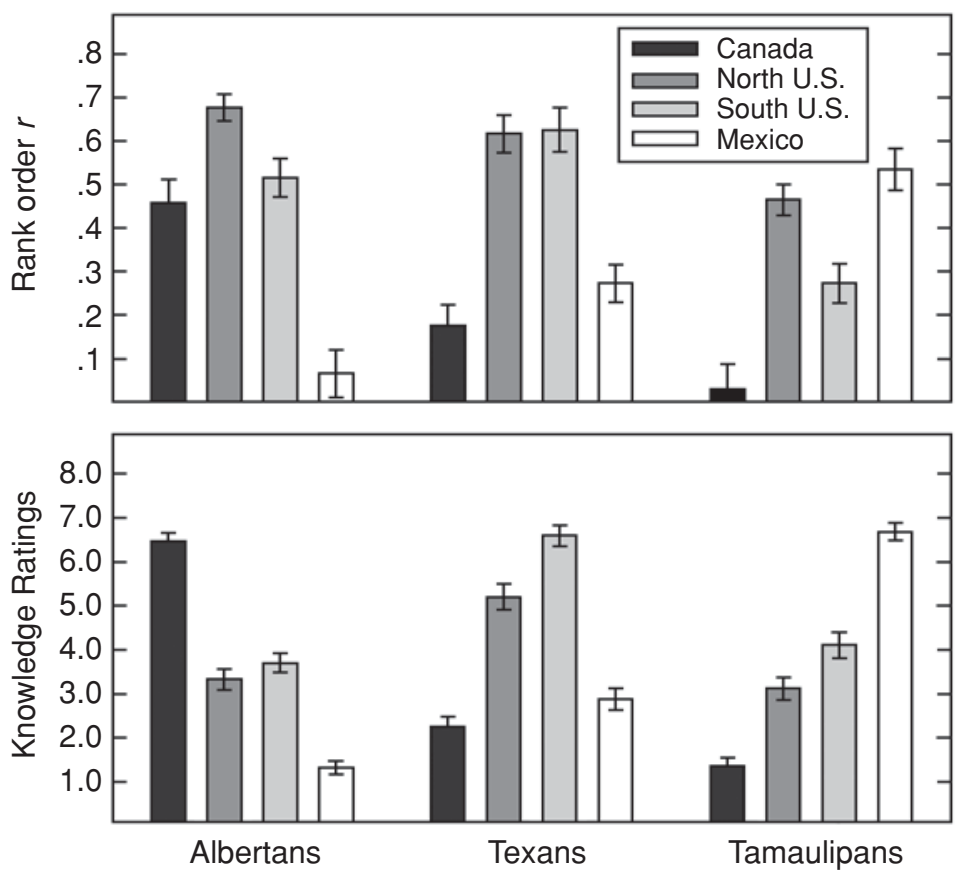

Figure 4. The top panel shows the average rank order correlations (back transformed from the mean Fisher values in the ANOVA), and the bottom panel shows the average knowledge ratings for each participant group and region. Error bars are standard errors of the mean for each region, computed across participants.

their knowledge of cities in their own regions the highest; the Albertans and Tamaulipans rated their knowledge of cities in the U.S. the next highest. The different patterns of ratings yielded a group $\times$ region interaction $[F(6,402)=$ 344.93, $\left.M S_{\mathrm{e}}=0.82\right]$. However, rated knowledge did not predict the specific pattern of either item or regional accuracy for any group.

\section{DISCUSSION}

Three novel findings arose from comparing the three groups of participants. First, the participants in all three groups were the least accurate in their estimates of the absolute location of Mexican cities, and they shared a propensity for placing most of them near the equator. Related to this was the finding that all three groups had a larger mean difference between their estimates for the locations of U.S. and Mexican cities than they did between the U.S. and Canadian cities; an examination of the proportional distances between regional means confirmed that the observed differences between them were a result of "stretching" the North American continent southward.

Because the knowledge rating task occurred first, the participants knew which cities were to be estimated and their respective countries. In addition, the instructions ensured that all three groups began with the same explicit values for the poles and the equator. The location estimate task thus involved deciding where, in the overall range of latitudes, these countries and any regions within them be- longed. That they all placed most Mexican cities near the equator, while maintaining the relative scale distances between regional means, argues forcefully that they shared the belief that the equator is at or near the southern boundary of North America or is the prototype for Mexico and that this belief was instrumental in defining the functional response range. Thus, shared beliefs about the locations of regions relative to global reference points was a key factor underlying the observed bias in global location estimates for all three groups.

The second novel finding in the present study was that despite the poor absolute accuracy in most of the estimates, all three groups showed good relative accuracy for the estimates in some regions; however, the regions that had relatively good rank order correlations differed among the groups. Third, North America was partitioned differently by the three groups.

By definition, cultural asymmetry is a construct that is knowledge based, so the different pattern of rank order correlations among the groups supports the idea that cultural asymmetry affected the mapping of relative locations within some regions. The differential partitioning of North America by the three groups also argues for the influence of culture-specific knowledge on the estimates. In particular, Albertans and Texans organized U.S. cities into two regions and Mexican cities into one, whereas the Tamaulipans organized U.S. cities into one region and Mexican cities into two. The two-region organization of U.S. cities might reflect Albertans' and Texans' knowledge about 
the U.S. Civil War, as well as well-known differences in climate between the regions. Similarly, the two-region organization of Mexican cities probably reflects the Tamaulipans' historical and geographical knowledge: Two of the three stimulus cities placed in northern Mexico (Tijuana and Ciudad Juarez) are border cities, which, together with Cabo San Lucas (a relatively recently developed resort), are considered agringada (heavily influenced by U.S. culture). By contrast, the three other resorts in the stimulus set (Acapulco, Mazatlán, and Puerto Vallarta) have a long prior history. In addition, between the major cities of northern Mexico and the Valley of Mexico in central/ southern Mexico is a relatively underpopulated, semiarid region. Finally, there is a difference between northern and southern Mexicans in their sense of belonging and in their participation in various cultural institutions (Cappello, 1996, 1998). Any one of these factors, or a combination, could have contributed to the two-region organization of Mexico by Mexican participants. This analysis predicts that participants from other countries with a history of regional divisiveness or distinctiveness (e.g., Great Britain, Italy, or Spain) would also produce estimates that revealed a multiregional knowledge organization.

Although the three findings just discussed are novel, they replicate and extend our previous work (Friedman, Kerkman, \& Brown, 2002; Kerkman, Friedman, Brown, Stea, \& Carmichael, 2003) and allow us to make stronger generalizations. In particular, the biases observed in the location estimates for most of the cities were related to mistaken beliefs about the locations of their superordinate regions, which in turn were related to mistaken beliefs about the boundaries for North America and the placement of the regions relative to these and to each other. Thus, the influence of regions on the estimates, and of the equator as a lower bound, seems to be shared among all three groups. In contrast, the assignment of cities to regions and their relative rank ordering reflected asymmetries in culture-specific knowledge.

In general, the data from the present study support the assumption that the pattern of global location estimates and the particular biases observed within and across cultures are multiply determined. Furthermore, they indicate that the location estimate paradigm can reveal aspects of representation and process in geographical cognition that are universal, as well as those that are culturally distinctive. As such, the paradigm can contribute to our understanding of how culture affects cognition.

\section{REFERENCES}

Bailenson, J. N., Shum, M. S., Atran, S., Medin, D. L., \& Coley, J. D. (2002). A bird's eye view: Biological categorization and reasoning within and across cultures. Cognition, 84, 1-53.

BRown, N. R. (2002). Real-world estimation: Estimation modes and seeding effects. In B. H. Ross (Ed.), The psychology of learning and motivation (Vol. 41, pp. 321-359). New York: Academic Press.

Brown, N. R., \& Siegler, R. S. (1993). Metrics and mappings: A framework for understanding real-world quantitative estimation. Psychological Review, 100, 511-534.
Cappello, H. M. (1996). National conscience along the northern Mexican border. International Journal of Social Sciences \& Humanities, 6, 27-44.

CApPello, H. M. (1998). National identity and civic-political character in two regions of Mexico: Comparison between north and southcenter cities. International Journal of Social Sciences \& Humanities, 8, 75-96.

Collins, A., \& Michalski, R. (1989). The logic of plausible reasoning: A core theory. Cognitive Science, 13, 1-49.

Friedman, A., \& Brown, N. R. (2000a). Reasoning about geography. Journal of Experimental Psychology: General, 129, 193-219.

FrIEdMAN, A., \& BROWN, N. R. (2000b). Updating geographical knowledge: Principles of coherence and inertia. Journal of Experimental Psychology: Learning, Memory, \& Cognition, 26, 900-914.

Friedman, A., Brown, N. R., \& McGaffey, A. P. (2002). A basis for bias in geographical judgments. Psychonomic Bulletin \& Review, 9, 151-159.

Friedman, A., Kerkman, D. D., \& Brown, N. R. (2002). Spatial location judgments: A cross-national comparison of estimation bias in subjective North American geography. Psychonomic Bulletin \& Review, 9, 615-623.

Golledge, R. G. (1993). Geographical perspectives on spatial cognition. In T. Gärling \& R. G. Golledge (Eds.), Behavior and environment: Psychological and geographical approaches (Advances in Psychology, Vol. 96, pp. 16-46). Amsterdam: North-Holland.

Huttenlocher, J., Hedges, L. V., \& Duncan, S. (1991). Categories and particulars: Prototype effects in estimating spatial location. Psychological Review, 98, 352-376.

Kerkman, D. D., Friedman, A., Brown, N. R., Stea, D., \& CarMiCHAEL, A. (2003). The development of geographic categories and biases. Journal of Experimental Child Psychology, 84, 265-285.

LundBERG, U., \& EKMAN, G. (1973). Subjective geographic distance: A multidimensional comparison. Psychometrika, 38, 113-121.

McNamara, T. (1986). Mental representations of spatial relations. Cognitive Psychology, 18, 87-121.

MCNAMARA, T., \& DiwAdKaR, V. A. (1997). Symmetry and asymmetry of human spatial memory. Cognitive Psychology, 34, 160-190.

Medin, D. L., Ross, N., Atran, S., Burnett, R. C., \& Blok, S. V. (2002). Categorization and reasoning in relation to culture and expertise. In B. H. Ross (Ed.), The psychology of learning and motivation: Advances in research and theory (Vol. 41, pp. 1-41). New York: Academic Press.

National Geographic Roper 2002 Global Geographic Survey (2002). Washington, DC: National Geographic Education Foundation. Stevens, A., \& Coupe, P. (1978). Distortions in judged spatial relations. Cognitive Psychology, 10, 422-437.

\section{NOTES}

1. The instructions were translated from English into Spanish by a Spanish-English bilingual speaker whose first language was Spanish. The Spanish translation was then back-translated into English by a bilingual speaker whose first language was English.

2. The Albertans were likely to be aware that their home city (Edmonton) was the most northern in the set. In addition, because the title of a popular Canadian television show about the Northwest Territories is "North of 60 ," the Albertans were probably also aware that the southern latitude of the Northwest Territories and, thus, the northern boundary of Alberta is $60^{\circ}$.

3 . We are grateful to an anonymous reviewer for suggesting this possibility.

4. We used all four regions in the rank order correlations, because the two U.S. regions together span a larger range of latitude than either the Mexican or the Canadian cities do; consequently, combining the two U.S. regions could lead to artifactually high rank order correlations.

(Manuscript received June 8, 2004; revision accepted for publication February 22, 2005.) 